\title{
Broadband Artificial Magnetic Conductors Constructed of Magnetically Coupled Elements
}

\author{
Pavel Petrov ${ }^{1}$, Alastair Hibbins ${ }^{1}$, Roy Sambles ${ }^{1}$ \\ ${ }^{1}$ University of Exeter, Department of Physics and Astronomy, EX4 4QL, Exeter, UK, pp386@exeter.ac.uk
}

\begin{abstract}
An Artificial Magnetic Conductor (AMC) is a type of metamaterial that can be used to enhance the performance in several antenna and microwave design applications. In this work we propose an analytical approach for size and bandwidth optimization of AMCs constructed of magnetically coupled elements Numerical results (obtained using COMSOL) for the optimized structure demonstrate performance close to the theoretical limit.
\end{abstract}

Index Terms-antennas, artificial magnetic conductors, helices, measurements.

\section{INTRODUCTION}

Artificial magnetic conductors (AMCs) have been first presented by Sievenpiper in 1999 as metallic arrays printed on a grounded dielectric substrate and connected to the ground plane through metal vias [1]. Such surfaces fully reflect for certain frequencies normally incident microwaves with a near zero degree reflection phase. Assuming no losses and exactly 0 reflection phase, the surface is referred to as a perfect magnetic conductor (PMC), complementary to a perfect electric conductor (PEC). In practice, the reflection phase of AMCs crosses zero at just one frequency (for one resonant mode). The useful bandwidth of an AMC is in general defined as the frequency band between phase changes on reflection of \pm 90 on either side of the central frequency, since these phase values would not cause destructive interference between direct and reflected waves. A schematic of such an AMC is shown on Fig. 1.

AMC surfaces have also been produced from structures similar to the Sievenpiper but without vias, which eases fabrication [2], [3]. Theoretical analysis of such structures can be found in [4].

Metallic arrays have also been placed over a ground plane in order to significantly enhance the directivity of simple radiating sources positioned between the array and the ground [5]-[7]. The structure is based on the formation of a resonant cavity between the ground plane and the array that acts as a partially reflective surface (PRS). While high gain planar antenna designs have been produced, the antenna profile, which is determined by the resonance condition of the cavity, has always been close to half wavelength.

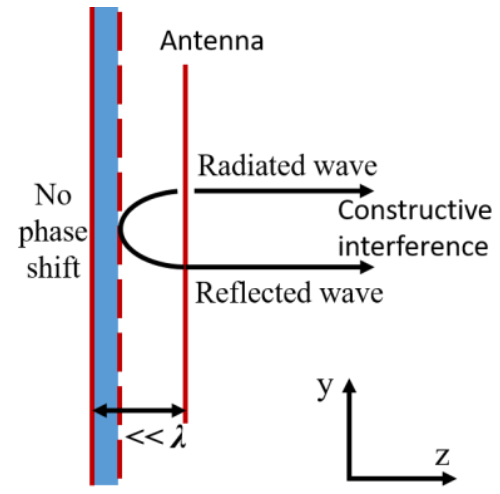

FIG. 1. Schematic representation of an antenna incorporated with an AMC consisting of an array of metallic patches placed above a ground plane.

Further work has been presented [8] where the antenna profile has been reduced to approximately half, maintaining the gain performance using planar periodic metallic arrays placed on a grounded dielectric substrate. There are further studies presented in other articles [9-14]. Depending on the intended application AMCs can be optimised in terms of their size relative to the wavelength in the radiation direction and their operational band. The previously published results provide a wide range of possible parameters with operational band ranging from $10 \%$ to $40 \%$ compared to the resonant frequency while the thickness varies from $5 \%$ to $7 \%$ of the wavelength [14]. It has been recently demonstrated that AMCs can be constructed of magnetically coupled helices [15], and structures that provide 31\% operational band while comprising $5 \%$ of the wavelength have been suggested.

Theoretical limitations of AMCs have been studied in [16], where Brewitt-Tailor estimated the maximum operational bandwidth of an AMC as:

$$
\frac{\Delta f}{f} \leq \pi^{2} \frac{h}{\lambda^{\prime}}
$$

where $\Delta f$ is operational band of the AMC, $f$ - its central frequency, $h$ - thickness of the AMC, $\lambda$ - wavelength.

The performance enhancements that can be achieved with the introduction of an AMC include: excitation of additional resonant frequencies in antenna integrated structure [17], improvement of antenna's bandwidth and gain [18-19], increase of RFID detection [20] and specific absorption rates for medical body-area network application [21]. 
In this work we introduce an analytical approach for the optimisation of AMCs constructed of magnetically coupled elements. Further we demonstrate numerical results for the structures which provide performance close to the BrewittTailor limit.

\section{ANALYTICAL OPTIMISATION}

To analyse the AMC performance we shall first introduce a parameter that will characterise how close the performance of the AMC is to the Brewitt-Tailor limit. We suggest an AMC parameter $P$, which equals 1 when the limit is reached, of the following form:

$$
P=\frac{\Delta f \lambda}{\pi^{2} f h}
$$

To illustrate, how close the existing structures are to the theoretical limit, we demonstrate the performance of some of the previously suggested AMCs on Fig. 2. It can be seen, that for the patch structure the AMC parameter drops significantly when the relative thickness decreases, while the helical one gives an opportunity to increase $P$ while making the relative size smaller. However, no geometries with AMC parameter much above 0.6 have been suggested.

First let us consider an infinite uniform 2D array of helical elements arranged parallel to each other on top of a ground plane. Each helix is electrically isolated from the ground plane and all other helices. We assume that the helical wires are infinitely thin and the current in any particular helix is uniform. The parameters of the helices are: radius $r_{h e l}$ and length $l_{h e l}$, while the parameters of the unit cell are: height $h_{c}>2 r_{h e l}$ width $w_{c}>2 r_{h e l}$, length $l_{c}>l_{\text {hel }}$. The array is excited by a plane electromagnetic wave with magnetic field $H=H_{0} e^{-i \omega t}$ parallel to the axes of the helices.

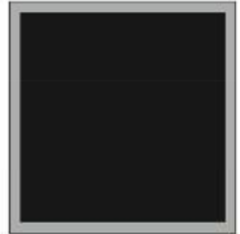

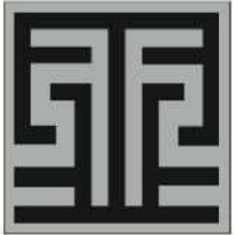

b

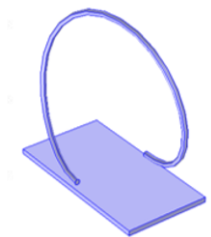

c

\begin{tabular}{|c|c|c|c|}
\hline Structure & $h / \lambda, \%$ & $\Delta f / f, \%$ & $P$ \\
\hline $\mathrm{a}$ & 7 & 40 & 0.58 \\
\hline $\mathrm{b}$ & 5.1 & 10 & 0.21 \\
\hline $\mathrm{c}$ & 5 & 30 & 0.61 \\
\hline
\end{tabular}

FIG. 2. Geometries of the AMC unit cells and their AMC performance for a) conventional patch structure [14], b) low frequency patch structure [14], and c) helical structure [15].

At operational frequencies an artificial magnetic conductor (AMC) should have a high value of surface impedance:

$$
Z=\sqrt{\frac{\mu}{\varepsilon}},
$$

Here $\mu$ is the effective relative magnetic permeability of the $\mathrm{AMC}$ and $\varepsilon$ is the effective relative electric permittivity. In the presented case the electromagnetic response of the helices will contribute a frequency dependence to $\mu$ while $\varepsilon$ will stay frequency independent to a first approximation. Therefore to obtain the best AMC response the $\mu$ of the structure has to be maximised over as wide a frequency range as possible.

The magnetic moment $m_{n m}$ of an individual helix positioned at row $n$ and column $m$ of the array $(n, m)$ can be calculated as that for a solenoid:

$$
m_{n m}=I_{n m} N S_{h e l},
$$

where $I_{n m}$ is the current in the helix, $N$ the number of turns and $S_{h e l}=4 \pi r_{h e l}$ the cross-section area of the helix. Kirchhoff's voltage equations for each element of the array can be written in the following form:

$$
I_{n m} Z_{n m}+\sum I_{a b} Z_{a b}=U,
$$

where $Z_{n m}$ is the complex impedance of the helix, $U$ the electromagnetic induction created by the oscillating magnetic field, $I_{a b}$ the current in the element positioned at $(\mathrm{n}+\mathrm{a}, \mathrm{m}+\mathrm{b})$ and $Z_{a b}$ and the mutual impedance of the elements is $(n, m)$ and $(n+a, m+b) . U$ can be expressed in the following way using Faraday's law:

$$
U=\mu_{0} N i \omega H S_{\text {hel }} \text {. }
$$

To calculate $I_{n m}$ we assume that as the infinite array is excited uniformly then the currents in all elements should be the same at any particular frequency and equal to $I$. In this case (5) can be rewritten in the following form:

$$
I\left(Z_{n m}+\sum Z_{a b}\right)=U,
$$

In order to obtain impedances we shall consider an helix as an equivalent LCR circuit, which is a valid assumption as the helices are significantly smaller than the wavelength at their first resonance frequency. Thus for the self-impedance we obtain:

$$
Z_{n m}=-i \omega L\left(\left(\frac{f_{0}}{f}\right)^{2}-1-i \frac{f_{0}}{f Q}\right),
$$

where $f_{0}$ is the resonance frequency of an individual helix, $Q$ its quality factor and $L=\mu_{0} S N^{2} / l_{\text {hel }}$ its self inductance, For the mutual-impedance:

$$
Z_{a b}=-i \omega L\left(\left(\frac{f_{0}}{f}\right)^{2}\left(\kappa_{E g r}+\kappa_{E a, b}\right)-\kappa_{H g r}-\sum_{a, b} \kappa_{H a, b}\right)
$$

where $\kappa_{\mathrm{Ea}, \mathrm{b}}$ and $\kappa_{\mathrm{Ha}, \mathrm{b}}$ are the electric and magnetic couplings between helices $(n, m)$ and $(n+a, m+b)$ respectively, $\kappa_{\text {Egr }}$ and $\kappa_{\mathrm{Hgr}}$ are the electric and magnetic couplings to the ground plane. More information on coupling coefficients can be found in [22] and the way to obtain the coupling values from numerical or experimental data is described in [23]. Now, substituting (6), (8) and (9) into (7) we obtain the equation for the current in each helix: 


$$
I=\frac{\mu_{0} N H S_{h e l}}{L\left(\left(\frac{f_{0}}{f}\right)^{2}-1-i \frac{f_{0}}{f Q}+\left(\frac{f_{0}}{f}\right)^{2}\left(\kappa_{E g r}+\sum_{a, b} \kappa_{E a, b}\right)-\kappa_{H g r}-\sum_{a, b} \kappa_{H a, b}\right)} .
$$

The magnetic moment of each element is:

$$
m=\frac{H V_{h e l}}{\left(\left(\frac{f_{0}}{f}\right)^{2}-1-i \frac{f_{0}}{f Q}+\left(\frac{f_{0}}{f}\right)^{2}\left(\kappa_{E g r}+\sum_{a, b} \kappa_{E a, b}\right)-\kappa_{H g r}-\sum_{a, b} \kappa_{H a, b}\right)},
$$

where $V_{\text {hel }}$ is the intrinsic volume of the helix. The relative magnetic permittivity can be expressed in the following form:

$$
\mu=1+\frac{4 \pi M}{H},
$$

$\mathrm{M}$ here is the magnetisation of our AMC. Finally, the expression for $\mu$ can be written as:

$$
\mu=1+\frac{4 \pi \alpha}{\left(\left(\frac{f_{0}}{f}\right)^{2}-1-i \frac{f_{0}}{f Q}+\left(\frac{f_{0}}{f}\right)^{2}\left(\kappa_{E g r}+\sum_{a, b} \kappa_{E a, b}\right)-\kappa_{H g r}-\sum_{a, b} \kappa_{H a, b}\right)},
$$

where $\alpha=V_{h e l} / V_{c}$ is the fill factor.

For further discussion we will assume that the helices are coupled to their nearest neighbours along both primary axes and also to their next nearest neighbours along the diagonal axes. Coupling to other helices will be neglected. In this case the coupling coefficients are non 0 for elements with $a$ and $b=-1,0,1$.

Now, to maximise the AMC response it is clear that the fill factor should be made as large as possible. Furthermore, magnetic coupling appears as a sum term in the denominator of (13). Thus it does not affect the relative operational width, but shifts the resonant frequency down, so strong positive magnetic coupling is required to obtain significant sub-wavelength response. The electric coupling coefficient is multiplied by $f_{0} / f$ and as a result strong electric coupling makes the resonance sharper which we would like to avoid. Finally the $Q$ factor can be made as small as possible to maximise the bandwidth. However it is also important to note that low $Q$ leads to high losses and decreases the resulting gain of the antenna when placed on top of the AMC.

\section{STRUCTURE OPTIMISATION}

As was shown in section 2, in order to maximise the AMC parameter of the structure with magnetic response, the elements should have relatively low Q and the fill factor should be close to 1 . Elements, that satisfy both of these requirements are split ring resonators shown on Fig. 3a. Square geometry has been chosen to provide maximum fill factor and allow manufacturing on double sided PCB. However, the electric coupling for this configuration is significant both between neighbouring elements and also to the ground plane. Thus, as can be seen in (13), apart from making the structure significantly subwavelength electric couplings also makes the resonance sharp, limiting the AMC parameters. The electric fields in this structure are concentrated in the gaps between pins and the ground plane.
To minimise electric coupling between elements, the pins have to be moved away from each other without decreasing the fill factor of the element. We propose doing this using the geometry shown on Fig. $3 \mathrm{~b}$ with pins moved by $l / 4$ from the centre of the top patch. This will also weaken the negative magnetic coupling from the nearest neighbours that will further increase the relative operational band. Furthermore to avoid the coupling from the diagonal elements, each second element can be mirrored (Fig. 3c). In this case the closest distance between pins corresponds to $l / 2$, which makes the electric coupling negligible.

Finally, to reduce coupling to the ground plane we suggest replacing the ground plane with the conductive grid shown on Fig. 3d. Despite the fact that this will lead to some transmission, the structure will still have a high reflection coefficient, while low electric coupling allows the more broadband response.
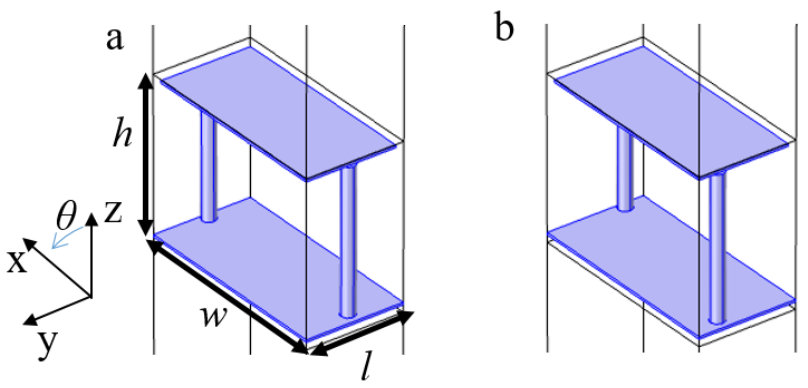

c
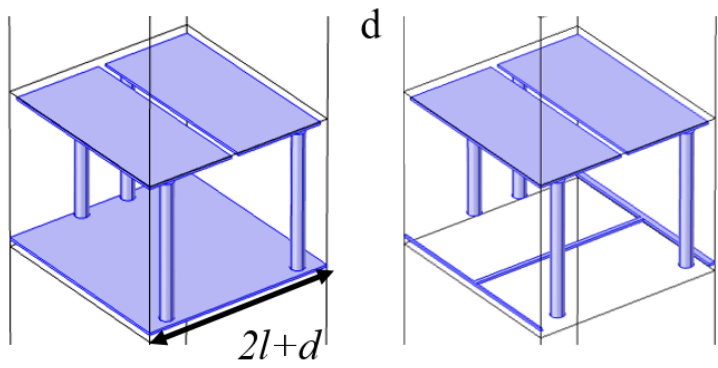

FIG. 3. Geometries of the AMC unit cells a) SRR on top of the ground plane, b) SRR with shifted pins on top of the ground plane, c) 2 mirrored SRRs on top of the ground plane d) 2 mirrored SRRs on top of the conductive grid.

\section{NUMERICAL RESULTS}

Numerical results presented in this section have been obtained using the frequency domain solver of the commercially available package COMSOL Multiphysics. The parameters of these structures are the following: $h=3$ $\mathrm{mm}, w=4 \mathrm{~mm}, l=2 \mathrm{~mm}$, distance between elements edges $d=0.15 \mathrm{~mm}$, spacing from ground plane to pins edge $g=$ $0.05 \mathrm{~mm}$, radius of pins $r=0.15 \mathrm{~mm}$. These values have been chosen to satisfy the current manufacturing capabilities. Materials used in the model are: copper for the conductive elements and FR4 with dielectric constant of 4.3 for the substance.

The frequency dependence of the phase difference between normally incident and reflected signal for all 
structures discussed in the previous section is presented on Fig. 4. Variations in central frequencies of the operational bands correspond to the discussed changes in coupling values. It can be seen that the relative operational band increases from $14 \%$ for structure (a) to $24 \%$ for structure (d).

The frequency dependence of the amplitude reflection coefficient is presented in Fig. 5. For the first three structures the losses are only associated with resistive losses and reach a maximum of about $5 \%$ at the central frequency. For the fourth structure, the drop in reflection coefficient arises from the reradiation from free pin edges that are arranged close to the conductive grid plane. The maximum drop is now $26 \%$ and is also observed at the central frequency. Away from the resonance the grid behaves like a ground plane as long as the holes in it are much smaller than the operational wavelength.

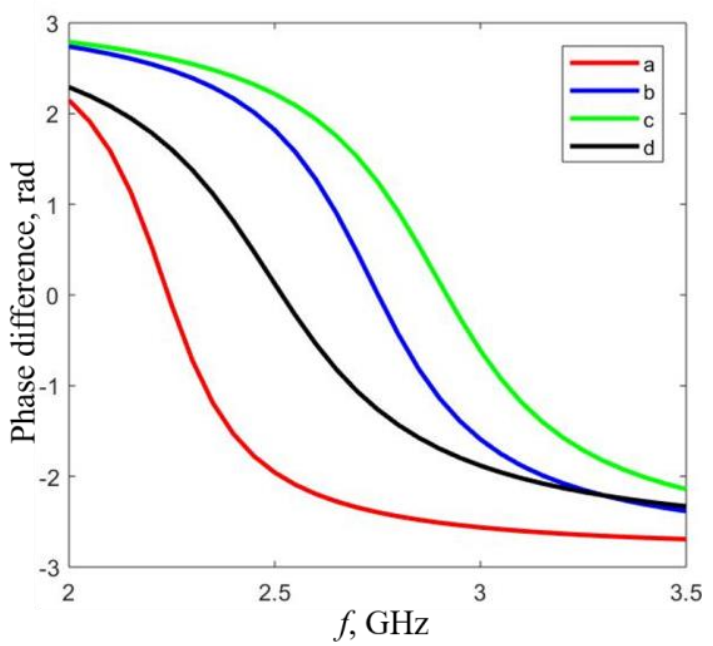

FIG. 4. Frequency dependence of the phase difference between normally incident and reflected waves for the structures presented on Fig.3.

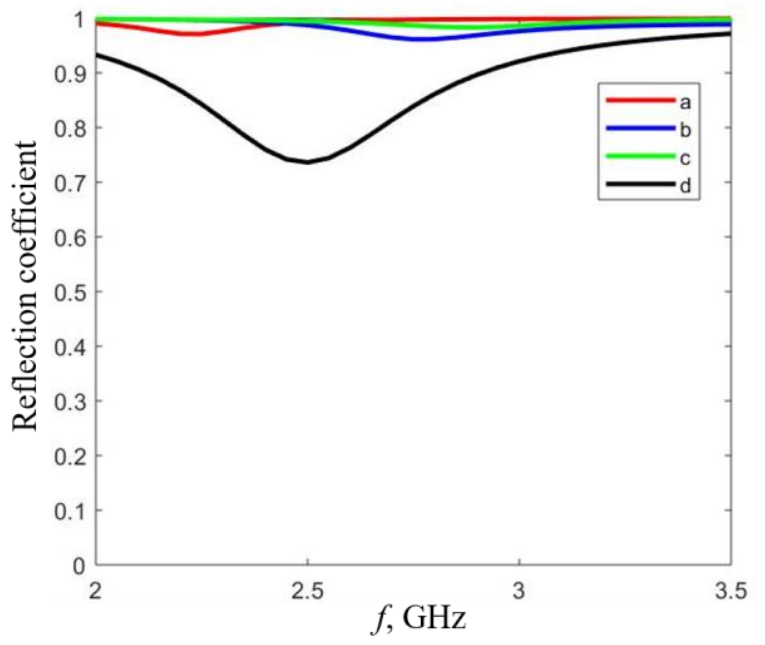

FIG. 5. Frequency dependence of the normally reflection coefficients for the structures presented on Fig.3.
The parameters for AMC performance for these geometries are shown in Table I. It can be seen that the AMC parameter increases for these structures with the decrease of electric coupling and a maximum of 0.96 is reached for the structure (d) that is close to the BrewittTailor limit. It is also important to note that all the presented structures are below $3 \%$ of the wavelength at the central frequency.

TABLE I. Parameters of AMCs. Structures presented in Fig. 3

\begin{tabular}{|c|c|c|c|}
\hline Structure & $h / \lambda, \%$ & $\Delta f / f, \%$ & $P$ \\
\hline $\mathrm{a}$ & 2.3 & 14 & 0.61 \\
\hline $\mathrm{b}$ & 2.7 & 17 & 0.63 \\
\hline $\mathrm{c}$ & 2.9 & 19 & 0.66 \\
\hline $\mathrm{d}$ & 2.5 & 24 & 0.96 \\
\hline
\end{tabular}

\section{CONCLUSIONS}

An analytic approach that can be used to optimise artificial magnetic conductors made of magnetically coupled elements has been introduced. Depending on the intended application the optimisation can be done in terms of bandwidth, relative size or the AMC parameter. This approach has been applied to the AMC structure constructed of split ring resonators placed on top of the ground plane and a close to optimal geometry have been suggested.

Numerical modelling of the optimised geometry demonstrates performance close to the Brewitt-Tailor limit with $24 \%$ operational bandwidth for the structure that comprises merely $2.5 \%$ of the wavelength at the central frequency.

Such structures can be manufactured on double sided PCBs and can be used in various microwave and antenna applications to improve the radiation performance and overcome the typical limitations in conventional antenna designs.

\section{ACKNOWLEDGEMENT}

We acknowledge financial support from the Engineering and Physical Sciences Research Council (EPSRC) of the United Kingdom via the EPSRC Centre for Doctoral Training in Metamaterials (Grant No. EP/L015331/1). We also acknowledge financial support from the Defence Science and Technology Laboratory (Dstl) (Contract No: DSTLX1000133579). All data created during this research are openly available from the University of Exeter's institutional repository at https://ore.exeter.ac.uk/.

\section{REFERENCES}

[1] D. Sievenpiper, L. Zhang, R. F. J. Broas, N. G. Alexopolous, and E.Yablonovitch, "High-impedance electromagnetic surfaces with a 
forbidden frequency band," IEEE Trans. Microw. Theory Tech., 47, pp. 2059-2074, 1999.

[2] Y. E. Erdemli, K. Sertel, R. A. Gilbert, D. E. Wright, and J. L. Volakis, "Frequency-selective surfaces to enhance performance of broad-band reconfigurable arrays," IEEE Trans. Antennas Propag., 50, pp. 1716-1724, 2002.

[3] Y. Zhang, J. von Hagen, M. Younis, C. Fischer, and W. Wiesbeck, "Planar artificial magnetic conductors and patch antennas," Special Issue on Metamaterials, IEEE Trans. Antennas Propag., 51, pp. 2704-2712, 2003

[4] W. E. McKinzie, and R. R. Fahr, "A low profile polarization diversity antenna built on an artificial magnetic conductor," In: IEEE Antennas and Propagation Society International Symposium, pp. 762-765, 2002.

[5] G. V. Trentini, "Partially reflecting sheet arrays," IRE Trans. Antennas Propag., AP-4, pp. 666-671, 1956.

[6] J. R. James, S. J. A. Kinany, P. D. Peel, and G. Andrasic, "Leakywave multiple dichroic beam formers," Electron. Lett., 25, pp. $1209-$ 1211, 1989.

[7] A. P. Feresidis and J. C. Vardaxoglou, "High gain planar antenna using optimized partially reflective surfaces," Proc. Inst. Elect. Eng. Microw. Antennas Propag., 148, pp. 345-350, 2001.

[8] A. P. Feresidis, G. Goussetis, S. Wang, and J. C. Vardaxoglou,"Artificial magnetic conductor surfaces and their application to low-profile high-gain planar antennas," IEEE Transactions on Antennas and Propagation, 53, pp 209-215, 2005.

[9] S. Wang, A. P. Feresidis, G. Goussetis, and J. C. Vardaxoglou,"Lowprofile resonant cavity antenna with artificial magnetic conductor ground plane," Electronics Letters, 40, pp. 405-406, 2004.

[10] S. H. Kim, T. T. Nguyen, and J. H. Jang, "Reflection characteristics of 1-D EBG ground plane and its application to a planar dipole antenna," Progress In Electromagnetics Research, 120, pp. 51-66, 2011.

[11] J. R. Sohn, K. Y. Kim, H.-S. Tae, and H. J. Lee, "Comparative study on various artificial magnetic conductors for low-profile antenna," Progress In Electromagnetics Research, 61, pp. 27-37, 2006.

[12] Y. Kim, F. Yang, and A. Z. Elsherbeni, "Compact artificial magnetic conductor designs using planar square spiral geometries," Progress In Electromagnetics Research, 77, pp. 43-54, 2007.

[13] K. Chung, J. Kim, and J. Choi, "Wideband microstrip-fed monopole antenna having frequency band-notch function," IEEE Microwave and Wireless Components Letters, 15, pp. 766-768, 2005.

[14] H. Oraizi, and R. Tirandaz, "Design of a novel miniaturized planar structure for the realization of artificial magnetic conductors", 23rd Iranian Conference on Electrical Engineering (ICEE), pp. 489-492, 2015.

[15] P. Petrov, A. Hibbins, I. Youngs, M. Lima, and R. Sambles, "Experimental Demonstration of Artificial Magnetic Conductors Constructed of Magnetically Coupled Helices," IEEE Proc. 14th European Conference on Antennas and Propagation (EuCAP), pp.1-5, 2020.

[16] C. R. Brewitt-Taylor, "Limitation on the bandwidth of artificial perfect magnetic conductor surfaces," IET Microw. Antennas Propag, 1, (1), pp 255-260, 2007

[17] M. F. Mohamadi, and N. Komjani, "Bandwidth enhancement of microstrip patch antenna using Jerusalem cross-shaped frequency selective surfaces by invasive weed optimization approach," Prog Electromagn Res., 121, pp. 103-120, 2011.

[18] B. S. Cook, and A. Shamim, "Utilizing wideband ame structures for high-gain inkjet-printed antennas on lossy paper substrate," IEEE Antennas Wirel Propag Lett., 12, pp. 76-79, 2013.

[19] S. Kim, R. Yu-Jiun, L. Hoseon, A. Rida, S. Nikolaou, and M.M. Tentzeri, "Monopole antenna with inkjet-printed ebg array on paper substrate for wearable applications," IEEE Antenn Wirel Propag Lett., 11, pp. 663-666, 2012

[20] I. Y. Park, and D. Kim, "Artificial magnetic conductor loaded long range passive RFID tag antenna mountable on metallic objects," Electron Lett. 50, pp. 335-336, 2014.

[21] H. R. Raad, A. I. Abbosh, H. M Al-Rizzo, and D. G. Rucker, "Flexible and compact amc based antenna for telemedicine applications," IEEE Trans Antenn Propag., 61, pp. 524-531, 2013.
[22] E. Tatartschuk, N. Gneiding, F. Hesmer, A. Radkovskaya and E. Shamonina, Mapping inter-element coupling in metamaterials: Scaling down to infrared, J. Appl. Phys. 111, 09490419, 2012.

[23] P. Petrov, A. Radkovskaya, and E. Shamonina: Retrieval of coupling coefficients for dense metamaterials, IEEE Proc. $10^{\text {th }}$ Int. Congress on Advanced Electromagnetic Materials in Microwaves and Optics, pp 283-285, 2016 